\title{
Pre-Operative Modified Glasgow Prognostic Score 2 For The Prediction of Poor Post-Operative Performance Status Following Palliative Surgery For Pathological Spinal Fracture: A Retrospective Cohort Study
}

Chikara Ushiku ( $\square$ chikara.u@gmail.com )

Tokyo Jikei University Kashiwa Hospital: Tokyo Jikeikai Ika Daigaku Fuzoku Kashiwa Byoin https://orcid.org/0000-0002-8456-0528

\section{Shoshi Akiyama}

Tokyo Jikei University Kashiwa Hospital: Tokyo Jikeikai Ika Daigaku Fuzoku Kashiwa Byoin

\section{Taku Ikegami}

Jikei University School of Medicine: Tokyo Jikeikai Ika Daigaku

\section{Takeshi Inoue}

Jikei University School of Medicine: Tokyo Jikeikai Ika Daigaku

\section{Akira Shinohara}

Jikei University School of Medicine: Tokyo Jikeikai Ika Daigaku

\section{Takayoshi Kajiwara}

Jikei University School of Medicine: Tokyo Jikeikai Ika Daigaku

\section{Daigo Arimura}

Jikei University School of Medicine: Tokyo Jikeikai Ika Daigaku

\section{Shunsuke Katsumi}

Jikei University School of Medicine: Tokyo Jikeikai Ika Daigaku

\section{Shintaro Obata}

Jikei University School of Medicine: Tokyo Jikeikai Ika Daigaku

\section{Shigeru Soshi}

Tokyo Jikei University Kashiwa Hospital: Tokyo Jikeikai Ika Daigaku Fuzoku Kashiwa Byoin

\section{Mitsuru Saito}

Jikei University School of Medicine: Tokyo Jikeikai Ika Daigaku

\section{Research article}

Keywords: Performance status, palliative surgery, pathological spinal fracture, modified Glasgow Prognostic Score, predictive factor 
Posted Date: July 19th, 2021

DOI: https://doi.org/10.21203/rs.3.rs-685550/v1

License: (c) (i) This work is licensed under a Creative Commons Attribution 4.0 International License. Read Full License 


\section{Abstract}

Background: Skeletal-related events due to spinal metastasis in cancer significantly impair patients' activities of daily living and quality of life. Most of these events occur suddenly. To reduce their impairment occurred suddenly, and to allow them to return to their normal life immediately, many patients undergo palliative surgery; however, some patients do not improve their performance status (PS) as expected. There is little evidence regarding the factors influencing a patient's PS after palliative surgery. We aimed to investigate the pre-operative predictors of poor PS 1 month after surgery.

Methods: The study included a consecutive series of 71 patients with pathological spinal fracture who underwent palliative surgery. Pre-operative predictors of poor post-operative PS were investigated. The participants were categorized into two groups according to PS; the Good group (PS 0, 1, or 2) and the Poor group (PS 3 or 4). We performed univariate and multivariable logistic regression analyses on demographic information, unidentified primary site, AIS grade, poor PS, spinal instability neoplastic score, revised Tokuhashi score, New Katagiri score, modified Glasgow Prognostic Score (mGPS), neutrophillymphocyte ratio(NLR), and prognostic nutrition index (PNI).

Results: Post-operatively, the Poor group included $38.0 \%$ of the patients. Univariate analysis revealed that the following pre-operative factors were related to poor outcomes $(p<0.05)$ : BMI<18.5; AIS grade $C$; poor PS; revised Tokuhashi score 0-8; New Katagiri score 7-10; mGPS 2; and PNI. In the multivariate analysis, mGPS $2(O R=22.8,95 \% \mathrm{Cl}=2.59-202.00, p<0.01)$ was a significant pre-operative predictor of poor postoperative PS.

Conclusion: mGPS 2 was a predictive clinical factor that influenced PS 1 month after surgery. Patients with mGPS 2 should be carefully evaluated to determine their treatment, especially whether they should undergo palliative surgery.

\section{Background}

Spinal metastasis is common in patients with advanced cancer. The reported incidence of spinal metastases is up to $60-70 \%$ at autopsy due to the prolonged life expectancy and advances in cancer therapy in recent decades, and $10-14 \%$ of patients develop symptomatic features and need surgical treatment with disease progression [1-7]. Skeletal-related events (SREs), such as pain and spinal paralysis, due to pathological fractures associated with spinal metastasis can significantly impair activities of daily living (ADLs) and quality of life (QOL) [8-13]. Impaired ADLs and QOL lead to delayed subsequent chemotherapy and even increased mortality [14]. Surgery can improve mechanical stability, and cord compression for spinal metastases is a palliative treatment aimed at improving patient QOL by alleviating pain and reversing or delaying neurological dysfunction, such as paraplegia and urinary incontinence. Many reports have described good outcomes of surgical intervention for metastatic spinal tumors in terms of neurological status and pain [15-21]. 
Although palliative surgical intervention for metastatic spinal tumors is generally favorable, some patients experience an unfavorable state with no improvement in ADLs and QOL. Overall, only $70-80 \%$ of patients achieve moderate or good general health as measured by the Karnofsky Index after surgery [16, 22], and only $80 \%$ of patients' families are satisfied or very satisfied with the surgical intervention [22, 23]. Although the patient's neurological condition or pain improves after the surgery, the patient's survival was the most important factor for satisfaction with the patient's family [23]. The survival of the patient depends on advancing primary cancer treatment. Several studies have shown that palliative chemotherapy, in general, does not prolong survival in patients with a poor Eastern Cooperative Oncology Group (ECOG) performance status (PS) due to a lower response rate and poor treatment tolerance [24, 25]. The American Society of Clinical Oncology (ASCO) advocates withholding palliative chemotherapy in patients with an ECOG PS of 3-4 (PS $\geq 3$ defined as "capable of only limited self-care, confined to bed or chair more than $50 \%$ of walking hours") and recommends best supportive care (BSC) instead [26, 27]. Smith and Hillner [28] suggested a simple rule that patients must be well enough to walk unaided into the clinic to receive chemotherapy. Therefore, the ability to improve PS is a critical issue for patients with spinal metastases and pathological fractures as a poor PS prevents them from receiving treatment for cancer. Even if patients with impaired ADLs or QOL due to SREs undergo surgery in the hope that PS will improve in order to receive treatment for the primary tumor, patients with an ECOG PS of 3-4 after surgery may not be suitable for potentially life-prolonging chemotherapy, which may be a disastrous problem for patients. In the case of spinal metastases or pathological fractures identified in the case of untreated primary tumors or in the early stages of treatment, it is critical to improve PS aggravated by pain and paralysis in order to continue treatment of the primary tumor. Given the nature of cancer, PS after intervention for spinal metastases or pathological fracture is an important determinant of subsequent treatment of the primary tumor and the patient as a whole; the timing of this decision should be relatively early after surgery. Since pain alone has the potential to be relieved by drugs and radiotherapy, in addition to paralysis in case of radiotherapy $[29,30]$, it is considered very important for medical professionals who perform palliative surgical treatment for spinal metastases to know the limitations of PS improvement in order to support the end of life of cancer patients.

Whether PS suddenly lost due to SRE can be immediately recovered by the palliative surgery is an important question, as is the availability of chemotherapy that can prolong survival. There is little evidence regarding the factors associated with poor PS relatively early after palliative surgery. Therefore, in this study, we aimed to investigate the pre-operative predictors for a poor post-operative PS 1 month after surgery for pathological fractures associated with spinal metastasis, limited to thoracic and lumbar spine levels to rule out the effects of upper limb function.

\section{Methods}

\section{Study design and participants}

This was a single-center retrospective study. Our hospital is a cancer medical treatment cooperation base hospital with 402 locations nationwide in Japan, which provide specialized cancer care. We 
retrospectively analyzed 71 consecutive patients with pathological fractures in thoracic and lumbar spine levels associated with spinal metastasis who underwent palliative surgery between May 2010 and March 2021 and who met the criteria described below. The purpose of the surgery was palliative, to improve ADLs and QOL by improving neurological deficits, preserving ambulatory function, and reducing pain. Referring to the report of Hikata et al. [31], indications for surgery in patients with spinal metastasis were: (1) presence of progressive paralysis and (2) intolerable neck, back, upper and lower limbs pain, due to compression by tumor to the spinal cord or cauda equina, and instability of pathological fracture. Patients underwent Balloon Kyphoplasty were excluded from this study. Patients with a single lesion of spinal metastasis who underwent total spondylectomy for curative treatment were also excluded. Three patients underwent laminectomy alone, and 39 patients underwent posterior stabilization alone. In the case of the lower thoracic spine, posterior stabilization alone was performed with the percutaneous pedicle screw system. The other 29 patients underwent a combination of laminectomy and posterior stabilization. There were six complications among the 71 patients. Overall, the complication rate was $8.5 \%$. Of the three patients who developed pneumonia, two had pulmonary embolism and one had delayed wound healing. The follow-up duration was at least 1 month after surgery. The American Spinal Injury Association Impairment Scale (AIS) grade [32] and ECOG PS [33] were used as the main variables for evaluating paralysis and ADLs. The AIS grade and ECOG PS were evaluated by two or more orthopedic surgeons, including a certified spinal educator (certified by the Japanese Society for Spine Surgery and Related Research).

\section{Data collection}

Age, sex, body mass index(BMI), primary tumor, distribution of spinal lesions of metastasis, AIS grade, spinal instability neoplastic score (SINS) [34], revised Tokuhashi score [18], New Katagiri score [35], modified Glasgow Prognostic Score (mGPS) [36], neutrophil-lymphocyte ratio (NLR), and prognostic nutrition index (PNI) [37] were collected for each participant just before surgery. The participants who recovered to AIS grade D or E were able to walk, while AIS A or B patients were bedridden. The participants were categorized into three groups: AIS grade A or B, AIS grade C, and AIS grade D or E. SINS was assessed for evaluating spinal instability.

The revised Tokuhashi score and New Katagiri score were used to assess the severity of spinal metastasis. All blood samples were collected, and the laboratory measurements of serum values of lymphocyte count, neutrophil count, c-reactive protein (CRP), and albumin were performed just before the operation. Patients who had both a serum elevation of CRP $(>1.0 \mathrm{mg} / \mathrm{dL})$ and hypoalbuminemia $(<3.5$ $\mathrm{g} / \mathrm{dL}$ ) were allocated an mGPS of 2 . Patients with only one of the abnormal values were allocated an mGPS of 1, and patients who had neither were allocated an mGPS of 0 [36]. The NLR was defined as the absolute neutrophil count divided by the absolute lymphocyte count. PNI was calculated using the following equation: $\mathrm{PNI}=(10 \times$ serum albumin $[\mathrm{g} / \mathrm{dL}])+(0.005 \times$ total lymphocytes $[1,000 / \mu \mathrm{L}])[37]$. PS was collected for each participant both immediately before surgery and 1 month after surgery. Concerning the degree of PS, the participants were categorized into two groups; the Good group (PS 0, 1, or 2) and the Poor group (PS 3 or 4). Medical history was surveyed from interviews with individuals, family members, and entries in the prescription record. 


\section{Statistical analysis}

To compare the relative pre-operative predictors for post-operative poor PS, multivariate logistic regression analyses were performed using EZR (Saitama Medical Center, Jichi Medical University, Saitama, Japan), which is a graphical user interface for $\mathrm{R}$ (The R Foundation for Statistical Computing, Vienna, Austria) [38]. The factors included in the multivariate model were age, sex, and other variables with a value of $p<0.05$ in the univariate analyses $(B M I<18.5$, AIS grade $C$, poor PS, revised Tokuhashi score $0-8$, and New Katagiri score $7-10$, mGPS 2, PNI). In the case of categorical data, only the positive Odds ratios (ORs) was used for multivariate analysis. Therefore, factors with an OR of less than 1 (AIS grade D or E, Tokuhashi score 9-11, New Katagiri score 4-6) were not employed in the multivariate analysis. A value of $p<0.01$ was considered statistically significant.

\section{Results}

\section{Demographic data}

The clinical features of all 71 patients are listed in Table 1. The participants, 50 males and 21 females, had a mean age of 66.8 years (range $34-87$ ). Of the 71 subjects, 28 had previously unidentified primary sites when spinal metastasis was found. Biopsies were performed in the 28 cases where the primary site could not be immediately identified when spinal metastases were found; the primary site was then identified in all but one case (Table 1). The most common primary cancer was in the lung (25.4\%), accounting for 18 cases. Other primary malignant tumor diagnoses included cancers such as prostate cancer, kidney cancer, lymphoma, uterus cancer, breast cancer, and colon cancer (Table 2).

\section{Comparison of pre- and post-operative paralysis and performance status}

The distribution of AIS grade C decreased from 32.4-15.5\%, and while that of AIS grades D and E significantly improved from $57.7-76.1 \%$, indicating that more patients were able to walk with improved paralysis 1 month after surgery. In the comparison of pre- and post-operative PS, the distribution of PS 0 , 1 , or 2 significantly improved from $26.8-62.0 \%$. On the contrary, the that of PS 3 or 4 significantly decreased from $73.2-38.0 \%$ (Table 3). Approximately one-third of all patients were found to have poor PS despite having undergone surgery.

\section{Univariate analysis to identify pre-operative predictors for poor post-operative performance status}

Treatment was considered successful; however, there were a certain number of cases where PS did not improve. Therefore, we investigated the pre-operative features of patients who did not achieve PS improvement. We performed a univariate analysis to explore the predictors related to poor post-operative PS. According to univariate analyses, BMI $<18.5$, pre-operative AIS grade C, poor PS, revised Tokuhashi 
score 0 - 8, New Katagiri score 7 - 10, mGPS 2 and PNI were identified as potential factors affecting poor post-operative PS (Table 4).

\section{Independent pre-operative predictors evaluated by multivariable logistic analysis}

Based on the results of the univariate analyses, we then performed multivariable logistic regression analyses using the factors that yielded a $\mathrm{p}<0.01$, in addition to age and gender, to determine the independent pre-operative predictors associated with poor post-operative PS. As shown in Table 5, multivariate regression analysis revealed that only mGPS $2(O R=22.8,95 \%$ confidence intervals $=2.59-$ 202.00, $\mathrm{p}<0.01$ ) were significant pre-operative predictors for poor post-operative PS.

\section{Discussion}

In the present study, we demonstrated the pre-operative predictors that could predict poor post-operative PS at 1 month after palliative surgery for pathological fractures associated with spinal metastasis patients limited to thoracic and lumbar spine levels to rule out the effects of upper limb function by multivariable logistic analysis with statistical adjustment for confounding factors. Only mGPS 2 was identified as pre-operative predictor for poor post-operative PS.

PS is an important tool used by oncology healthcare professionals to assess the fitness of patients for systemic anticancer therapy and to predict prognosis in advanced malignancy. PS of spinal metastasis usually improves by 1 month after palliative surgery $[39,40]$. Similarly, in this study, the number of patients with poor PS (3-4) decreased significantly 1 month after surgery. However, $38.0 \%$ of patients still had poor PS 1 month after surgery in this study. Yamashita et al. reported that the PS outcomes 1 month after palliative surgery for spinal metastases depended on the pre-operative revised Tokuhashi score [41]. Reports indicate that pre-operative poor PS were significant independent prognostic factors associated with poor survival in patients with spinal metastases after palliative surgery [42], and the ambulatory functional outcomes after metastatic spinal tumor surgery were also influenced by the preoperative PS [43].

In contrast to previous reports, pre-operative mGPS 2, which has rarely been considered, was found to be a pre-operative predictor of poor post-operative PS 1 month after surgery in this study. The systemic inflammatory response plays an important role as it is an important cause of energy imbalance and muscle wasting cancer cachexia [44]. The production of pro-inflammatory cytokines triggers systemic inflammation with elevated CRP and decreased albumin levels [45]. The most widely accepted index for specialization of systemic inflammation is the mGPS [36]. This score combines two simple clinically available biomarkers (CRP and albumin) and has been employed in many different cancer patients. Furthermore, it was previously shown to be associated with prognosis in advanced cancers and is recognized as an identifier of cancer cachexia [46]. Cancer cachexia is a multifactorial syndrome that results in a decrease in skeletal muscle mass with or without a decrease of fat mass. Skeletal muscle wear associated with cancer cachexia is clinically important because it contributes to the reduction or 
discontinuation of cancer treatment and is associated with increased morbidity and mortality [47-50]. Muscle function may be directly proportional to muscle mass, and a decrease in muscle mass can lead to muscle weakness and loss of function, which adversely affect QOL [47, 50-52]. From the results of using pre-operative mGPS as a pre-operative predictor, it appears that PS may not improve due to loss of muscle mass. This implies that sarcopenia and other problems associated with cancer cachexia may not improve, even if there is no paralysis. While surgery for spinal metastasis improves pain and paralysis and improves ADLs and QOL in many cases, surgery may not improve PS in some cases, and there may be hesitation to perform surgical interventions. Spinal metastasis should have a higher priority, and immediate intervention should occur before the development of irreversible neurological deficits. Moreover, short-course radiotherapy and/or pain control with opioids and other medications may be good options for patients with a limited life span, especially in patients with mGPS 2.

There were several limitations to the present study. First, this was a retrospective observational study; therefore, the level of evidence was confined. Second, the sample size was small $(n=71)$. The relatively small sample size and the possibility of selection bias might discredit this study to chance findings. Third, the degree of pain control was not evaluated for pain control in patients in the present study. An improvement in pain is seen by 6 weeks after surgery [53]. In our experience, most of the patients experienced pain relief 2 weeks after surgery, but since the assessment was not performed in this study, the effect of pain on PS remains unknown. A future comprehensive prospective study may resolve these limitations.

In conclusion, we investigated the predictive clinical factors associated with poor post-operative PS. In the multivariable analysis, mGPS 2 was found to be a predictive clinical factor influencing poor postoperative PS $(p<0.01)$. This deleterious pre-operative clinical finding is a useful early predictor of poor PS 1 month after palliative surgery. Patients with mGPS 2 should be carefully evaluated to determine whether they should undergo palliative surgery.

\section{Abbreviations}

ADL: Activities of daily living

ASCO: American Society of Clinical Oncology

AIS: American Spinal Injury Association Impairment Scale

BMl: Body mass index

BSC: Best supportive care

ECOG: Eastern Cooperative Oncology Group

mGPS: modified Glasgow Prognostic Score 
NLR: Neutrophil-lymphocyte ratio

PS: Performance status

PNI: Prognostic nutrition index

QOL: Quality of life

SREs: Skeletal-related events

SINS: Spinal instability neoplastic score

\section{Declarations}

Ethics approval and consent to participate: The study protocol was approved by the institutional review board. Informed consent was obtained from each patient. The study was conducted in accordance with the principles of the Declaration of Helsinki and with the laws and regulations of Japan.

Consent for publication: Not applicable

Availability of data and materials: All data used and analyzed during this study are available from the corresponding author on reasonable request.

Competing interests: The authors declare that they have no competing interests.

Funding: The authors did not receive support from any organization for the submitted work.

Authors' contributions: CU designed the research; CU, SA, TI, TI, AS, TK, DA, SK, SO and SS collected and analyzed the data; CU wrote the paper; MS supervised the study. The authors read and approved the final manuscript.

Acknowledgments: The authors thank all the staff at The Jikei University Kashiwa Hospital for their kind support.

\section{References}

1. Yáñez ML, Miller JJ, Batchelor TT. Diagnosis and treatment of epidural metastases. Cancer 2017;7:1106-1114 DOI: 10.1002/cncr.30521

2. Ciftdemir M, Kaya M, Selcuk E, et al. Tumors of the spine. World J Orthop 2016;7:109-116 DOI: 10.5312/wjo.v7.i2.109

3. Bartels RH, van der Linden YM, van der Graaf WT. Spinal extradural metastasis: review of current treatment options. CA Cancer J Clin 2008;58:245-259 DOI: 10.3322/CA.2007.0016 
4. Bollen L, van der Linden YM, Pondaag W, et al. Prognostic factors associated with survival in patients with symptomatic spinal bone metastases: a retrospective cohort study of 1,043 patients. Neuro Oncol 2014;16:991-998 DOI: 10.1093/neuonc/not318

5. Witham TF, Khavkin YA, Gallia GL, et al. Surgery insight: current management of epidural spinal cord compression from metastatic spine disease. Nat Clin Pract Neurol 2006;2:87-94 DOI:

10.1038/ncpneuro0116

6. Bartels RH, Feuth T, van der Maazen R, et al. Development of a model with which to predict the life expectancy of patients with spinal epidural metastasis. Cancer 2007;110:2042-2049 DOI:

10.1002/cncr.23002

7. Spratt DE, Beeler WH, de Moraes FY, et al. An integrated multidisciplinary algorithm for the management of spinal metastases: an International Spine Oncology Consortium report. Lancet Oncol 2017;18:e720-e730 DOI: 10.1016/S1470-2045(17)30612-5

8. Coleman RE. Metastatic bone disease: Clinical features, pathophysiology and treatment strategies. Cancer Treat Rev 2001;27:165-176 DOI: 10.1053/ctrv.2000.0210

9. Coleman RE. Skeletal complications of malignancy. Cancer 1997;80:1588-1594 DOI:

10.1002/(sici)1097-0142(19971015)80:8+<1588::aid-cncr9>3.3.co;2-z

10. Plunkett TA, Rubens RD. The biology and management of bone metastases. Crit Rev Oncol Hematol 1999;31:89-96 DOI: 10.1016/s1040-8428(99)00008-6

11. Norgaard M, Jensen AO, Jacobsen JB, et al. Skeletal related events, bone metastasis and survival of prostate cancer: A population based cohort study in Denmark (1999 to 2007). J Urol 2010;184:162-167 DOI: 10.1016/j.juro.2010.03.034

12. Yong M, Jensen AO, Jacobsen JB, et al. Survival in breast cancer patients with bone metastases and skeletal-related events: A population-based cohort study in Denmark (1999-2007). Breast Cancer Res Treat. 2011;129:495-503 DOI: 10.1007/s10549-011-1475-5

13. Sathiakumar N, Delzell E, Morrisey MA, et al. Mortality following bone metastasis and skeletal-related events among men with prostate cancer: A population-based analysis of us medicare beneficiaries, 1999-2006. Prostate Cancer Prostatic Dis 2011;14:177-183. DOI: 10.1038/pcan.2011.7

14. Eda H, Santo L, David Roodman G, et al. Bone disease in multiple myeloma. Cancer Treat Res 2016;169:251-70 DOI: 10.1007/978-3-319-40320-5_14

15. Choi D, Crockard A, Bunger C, et al. Review of metastatic spine tumour classification and indications for surgery: the consensus statement of the Global Spine Tumour Study Group. Eur Spine J 2010;19:215222. DOI: $10.1007 / \mathrm{s} 00586-009-1252-x$ 
16. Ibrahim A, Crockard A, Antonietti P, et al. Does spinal surgery improve the quality of life for those with extradural (spinal) osseous metastases? An international multicenter prospective observational study of 223 patients. Invited submission from the Joint Section Meeting on Disorders of the Spine and Peripheral Nerves, March 2007. J Neurosurg Spine 2008;8:271-278 DOI: 10.3171/SPI/2008/8/3/271

17. Tomita K, Kawahara N, Kobayashi T, et al. Surgical strategy for spinal metastases. Spine 2001;26:298-306 DOI: 10.1097/00007632-200102010-00016

18. Tokuhashi $\mathrm{Y}$, Matsuzaki $\mathrm{H}$, Oda $\mathrm{H}$, et al. A revised scoring system for preoperative evaluation of metastatic spine tumor prognosis. Spine 2005;30:2186-2191 DOI:

10.1097/01.brs.0000180401.06919.a5

19. Hatrick NC, Lucas JD, Timothy AR, et al. The surgical treatment of metastatic disease of the spine. Radiother Oncol 2000;56:335-339 DOI: 10.1016/s0167-8140(00)00199-7

20. Hussein AA, El-Karef E, Hafez M. Reconstructive surgery in spinal tumours. Eur J Surg Oncol 2001;27:196-199 DOI: 10.1053/ejso.2000.1079

21. Hirabayashi H, Ebara S, Kinoshita T, et al. Clinical outcome and survival after palliative surgery for spinal metastases: palliative surgery in spinal metastases. Cancer 2003;97:476-484 DOI:

10.1002/cncr.11039

22. Weigel B, Maghsudi M, Neumann C, et al. Surgical management of symptomatic spinal metastases. Postoperative outcome and quality of life. Spine 1999;24:2240-2246 DOI: 10.1097/00007632199911010-00012

23. Fujibayashi S, Neo M, Miyaki K, et al. The value of palliative surgery for metastatic spinal disease: satisfaction of patients and their families. Spine J 2010;1:42-49 DOI: 10.1016/j.spinee.2009.06.016

24. Wheatley-Price P, Ali M, Balchin K, Spencer J, et al. The role of palliative chemotherapy in hospitalized patients. Curr Oncol 2014;4:187-192 DOI: 10.3747/co.21.1989

25. Crosara TM, Marques DF, Ferrari AC, et al. The effects of palliative chemotherapy in metastatic colorectal cancer patients with an ECOG performance status of 3 and 4 . Clin Colorectal Cancer 2015;1:52-57 DOI: 10.1016/j.clcc.2014.09.010

26. Peppercorn JM, Smith TJ, Helft PR, et al. American Society of Clinical Oncology statement: toward individualized care for patients with advanced cancer. J Clin Oncol 2011;6:755-760 DOI:

$10.1200 / J C 0.2010 .33 .1744$

27. Schnipper LE, Smith TJ, Raghavan D, et al. American Society of Clinical Oncology identifies five key opportunities to improve care and reduce costs: the top five list for oncology. J Clin Oncol 2012;14:17151724 DOI: $10.1200 / J C 0.2012 .42 .8375$ 
28. Smith TJ, Hillner BE. Bending the cost curve in cancer care. N Engl J Med 2011;364:2060-2065 DOI: 10.1056/NEJMsb1013826

29. Ahmad I, Ahmed MM, Ahsraf MF, et al. Pain Management in Metastatic Bone Disease: A Literature Review. Cureus 2018;9:e3286 DOI: 10.7759/cureus.3286

30. Matsumura A, Hoshi M, Takami M, et al. Radiation Therapy without Surgery for Spinal Metastases: Clinical Outcome and Prognostic Factors Analysis for Pain Control. Global Spine J 2012;3:137-142 DOI: $10.1055 / \mathrm{s}-0032-1326948$

31. Hikata T, Isogai N, Shiono Y, et al. A Retrospective Cohort Study Comparing the Safety and Efficacy of Minimally Invasive Versus Open Surgical Techniques in the Treatment of Spinal Metastases. Clinical Spine Surgery 2017;8:E1082-87 DOI: 10.1097/BSD.0000000000000460

32. Maynard FM, Jr, Bracken MB, Creasey G, et al. International Standards for Neurological and Functional Classification of Spinal Cord Injury. American Spinal Injury Association. Spinal Cord 1997;35:266-274. DOI: 10.1038/sj.sc.3100432

33. Oken MM, Creech RH, Tormey DC, et al. Toxicity and response criteria of the Eastern Cooperative Oncology Group. Am J Clin Oncol 1982;5:649-655

34. Fisher CG, DiPaola CP, Ryken TC, et al. A novel classification system for spinal instability in neoplastic disease: an evidence-based approach and expert consensus from the Spine Oncology Study Group. Spine 2010;22:E1221-9 DOI: 10.1097/BRS.0b013e3181e16ae2

35. Katagiri $\mathrm{H}, \mathrm{Okada} \mathrm{R}$, Takagi T, et al. New Prognostic factors and scoring system for patients with skeletal metastasis. Cancer Medicine 2014;5:1359-1367 DOI: 10.1002/cam4.292

36. McMillan DC. An inflammation-based prognostic score and its role in the nutrition-based management of patients with cancer: nutrition Society and BAPEN Medical Symposium on 'Nutrition support in cancer therapy... Proc Nutr Soc 2008;67:257-262 DOI: 10.1017/S0029665108007131

37. Mohri Y, Inoue $Y$, Tanaka K, et al. Prognostic nutritional index predicts postoperative outcome in colorectal cancer. World J Surg 2013;37:2688-2692 DOI: 10.1007/s00268-013-2156-9

38. Kanda Y. Investigation of the freely available easy-to-use software 'EZR' for medical statistics. Bone Marrow Transpl 2013;48:452-458 DOI: 10.1038/bmt.2012.244

39. Kakutani K, Sakai Y, Maeno K, et al. Prospective Cohort Study of Performance Status and Activities of Daily Living After Surgery for Spinal Metastasis. Clin Spine Surg 2017;30:E1026-32 DOI:

10.1097/BSD.0000000000000456

40. Kanda Y, Kakutani K, Sakai Y, et al. Prospective cohort study of surgical outcome for spinal metastases in patients aged 70 years or older. Bone Joint J 2020;12:1709-1716 DOI: 10.1302/0301- 
41. Yamashita T, Aota $\mathrm{Y}, \mathrm{Kushida} \mathrm{K}$, et al. Changes in physical function after palliative surgery for metastatic spinal tumor: association of the revised Tokuhashi score with neurologic recovery. Spine 2008;21:2341-6 DOI: 10.1097/BRS.0b013e3181878733

42. Kobayashi K, Ando K, Nakashima H, et al. Prognostic Factors in the New Katagiri Scoring System after Palliative Surgery for Spinal Metastasis. Spine 2020;13:E813-E819 DOI:

$10.1097 /$ BRS. 0000000000003415

43. Moon KY, Chung CK, Jahng TA, et al. Postoperative survival and ambulatory outcome in metastatic spinal tumors: prognostic factor analysis. J Korean Neurosurg Soc 2011;3:216-23 DOI: 10.3340/jkns.2011.50.3.216

44. Lindenmann J, Fink-Neuboeck N, Koesslbacher M, et al. The influence of elevated levels of C-reactive protein and hypoalbuminemia on survival in patients with advanced inoperable esophageal cancer undergoing palliative treatment: CRP and albumin in esophageal cancer. J Surg Oncol 2014;110:645-50 DOI: $10.1002 /$ jso. 23711

45. Vanhoutte G, van de Wiel M, Wouters K, et al. Cachexia in cancer: what is in the definition? BMJ Open Gastroenterol 2016;3:e000097ＤOI: 10.1136/bmjgast-2016-000097

46. Douglas E, McMillan DC. Towards a simple objective framework for the investigation and treatment of cancer cachexia: the Glasgow Prognostic Score. Cancer Treat Rev 2014;40:685-91 DOI:

10.1016/j.ctrv.2013.11.007

47. Fearon K, Strasser F, Anker SD, et al. Definition and classification of cancer cachexia: an international consensus. Lancet Oncol 2011;12:489-95 DOI: 10.1016/S1470-2045(10)70218-7

48. Andreyev HJ, Norman AR, Oates $\mathrm{J}$, et al. Why do patients with weight loss have a worse outcome when undergoing chemotherapy for gastrointestinal malignancies? Eur J Cancer 1998;34:503-509. DOI: 10.1016/s0959-8049(97)10090-9

49. Attaix D, Ventadour $S$, Codran A, et al. The ubiquitin-proteasome system and skeletal muscle wasting. Essays Biochem 2005;41:173-186 DOI: 10.1042/EB0410173

50. Prado CM, Baracos VE, McCargar LJ, et al. Sarcopenia as a determinant of chemotherapy toxicity and time to tumor progression in metastatic breast cancer patientsreceivingcapecitabine treatment. Clin Cancer Res 2009;15:2920-2926 DOI: 10.1158/1078-0432.CCR-08-2242

51. Argiles JM, Busquets S, Stemmler B, et al. Cancer cachexia: understanding the molecular basis. Nat Rev Cancer 2014;14:754-762 DOI: 10.1038/nrc3829 
52. Muscaritoli M, Bossola M, Aversa Z, et al. Prevention and treatment of cancer cachexia: new insights into an old problem. Eur J Cancer 2006;42:31-41 DOI: 10.1016/j.ejca.2005.07.026

53. Falicov A, Fisher CG, Sparkes MJ, et al. Impact of surgical intervention on quality of life in patients with spinal metastases. Spine.2006;24:2849-2856 DOI: 10.1097/01.brs.0000245838.37817.40

\section{Tables}

Due to technical limitations, table $1,2,3,4 \& 5$ is only available as a download in the Supplemental Files section.

\section{Supplementary Files}

This is a list of supplementary files associated with this preprint. Click to download.

- Table171.xlsx

- Table271.xlsx

- Table371.xlsx

- Table471v3.xIsx

- Table571v2.xIsx 